\title{
TAHSP:-
}

The Internet Joutnal of Allied Health Sciences and Practice

A Peer Reviewed Publication of the College of Health Care Sciences at Nova Southeastern University

Dedicated to allied health professional practice and education

http://ijahsp.nova.edu Vol. 11 No. 2 ISSN 1540-580X

\section{The Effect of Pointe Shoe Toe Box Shape on Proprioception in Novice Ballet Dancers}

\author{
Robert Cox, MS, LAT, ATC1 \\ Valerie W. Herzog, EdD, LAT, ATC2
}

1. Head Athletic Trainer, Heber Valley Physical Therapy, Heber City, Utah

2. Graduate Athletic Training Program Director, Department of Health Promotion and Human Performance, Weber

State University, Ogden, Utah

\section{United States}

CITATION: Cox R, Herzog V. The Effect of Pointe Shoe Toe Box Shape on Proprioception in Novice Ballet Dancer. The Internet Journal of Allied Health Sciences and Practice. April 2013. Volume 11 Number 2.

\begin{abstract}
Purpose: The purpose of this study was to determine the short-term effect of a pointe shoe toe box shape on proprioception in novice ballet dancers. Methods: Thirty-six female subjects completed the study (age $=13.2+2.2$ years, height $=156.4+11.5$ $\mathrm{cm}$, weight $=46.6+9.4 \mathrm{~kg}$ ). Subjects were assigned randomly to one of two groups. One group of 18 subjects was assigned to the tapered toe box pointe shoe, and the other group of 18 subjects was assigned to the square toe box pointe shoe. Subjects in each group were given a choice of any variation, brand, or size of shoe, only being restricted on the tapered or square box style. They were expected to train using that shoe, exclusively, over the next six weeks. Each subject's static proprioception (the Center of Pressure [COP] area [Area95] and reaction velocity [Vavg]) was measured prior to and following their six weeks of standard training using the AMTI AccuSway Plus forceplate. Results: A mixed-design ANOVA showed no effect on static proprioception for either shoe type. Conclusions: The shape of pointe shoe toe boxes does not have an effect on static proprioception in novice ballet dancers in a six week training program. Future research should evaluate these effects over a longer duration to evaluate long-term effects.
\end{abstract}

\section{BACKGROUND}

Like competitive athletes in traditional sports, ballet dancers must perform in environments that are demanding and pose a risk of physical injury. However, there are many tasks and strains placed upon dancers that differ from those placed on a typical athlete. ${ }^{1}$ Dancers have to be in the same level of condition as a highly trained athlete to perform, yet because ballet is also a performance art, there are fewer limitations or rules designed for maintaining the safety of the dancer. In the performing arts, quality is more than just the height of a jump, but is also judged by aesthetics of the performance. ${ }^{2}$ One aspect of aesthetics is the pointe shoe worn in ballet, which is intended to create a graceful line from the toe to the hip. However, it may be responsible for lower extremity injuries in ballet dancers, particularly in the foot and ankle, as the pointe shoe requires the placement of the full weight of the body on the toes for extended periods of time. ${ }^{1}$

Though many types of injuries may result from the use of pointe shoes, this study only addressed the issue of proprioception, which may be an underlying cause of several injuries. Proprioception is simply coordination or the awareness of the body and its limbs and their location as they move through space. It functions through a reflex arc, where afferent nerves communicate changes in the environment, such as air pressure changes, touch, and other senses, and the efferent motor neurons then act to make adjustments. ${ }^{3}$ In balance, the feet sense changes in pressure from the ground, shifting the center of pressure (COP), and the motor neurons then activate the appropriate muscles to compensate and avoid falling. Proprioception is, therefore, learned through practice, and it is dependent upon factors such as muscle strength and fiber types, flexibility, motor units, and muscle 
chains and memory. If any of these systems are lacking or are impeded by outside restrictions, then the body is at greater risk of injury.4-7 If injury does not happen, and if restrictions are continuously applied, then whatever system is impeded may weaken due to the lack of use. For example, if certain postural muscles in the foot are inhibited due to prolonged constriction by a narrow shoe, atrophy will likely develop.

Kinesthesia, or the sensation of joint positioning and movement, can in most cases only be sensed by the somato-senses, and it is often corrected by the simple reflex arc. ${ }^{5}$ As the afferent nerve ending's specialized mechanoreceptors provide input to the central nervous system (CNS) concerning external forces, reflex muscle contractions around the joint activate and adapt to control sudden movement acceleration or deceleration. This segmental feedback system, or reflex arc, which is generally governed by Renshaw cells in the spinal cord rather than the brain, can work with muscle spindles and golgi tendon organs in order to subconsciously maintain a controlled balance, even during locomotion..$^{6-7}$ This proprioceptive position sense is also known as Postural Control (PC).8-12

Postural control requires more than just appropriately developed neural pathways. Flexibility is required for a muscle to be functional in a full range of motion. Muscular strength has been shown to have a significant effect on proprioception. ${ }^{13}$ Strength gains typically result in improvements in proprioception, even in the absence of balance training. Muscle weakness, in turn, has been shown to decrease proprioception, which can result in injury. ${ }^{14}$ Muscle memory then ties these systems together through muscle chains involving full motor units and the muscle spindle reflex arc. As various motor neurons are activated repeatedly, more dendrites are created, and other motor units are incorporated to work synergistically. This results in greater force production and coordinated movements. All of these mechanisms work together to create what is sometimes referred to as muscle memory, the ideal unit for a reflex arc to correct changes in pressure and adapt to the surrounding environment. ${ }^{15-18}$

One major impedance of proprioception in pointe shoes may come from the toe box. The toe box is the tip of the shoe, and is generally made with wood or glued cardboard, or in some modern cases, plastic. The case is designed to hold toes together in order for them to support each other. Ideally, it is also designed to support all phalangeal joints, extending from the distal phalange to the metatarsophalangeal joint of the foot. This box also supports and protects the joints from giving way under the strain of body weight (see Figure 1).

Figure 1.19 This Shows the Box and Shank as they are Designed to Support the Structures and Alignment of the Foot

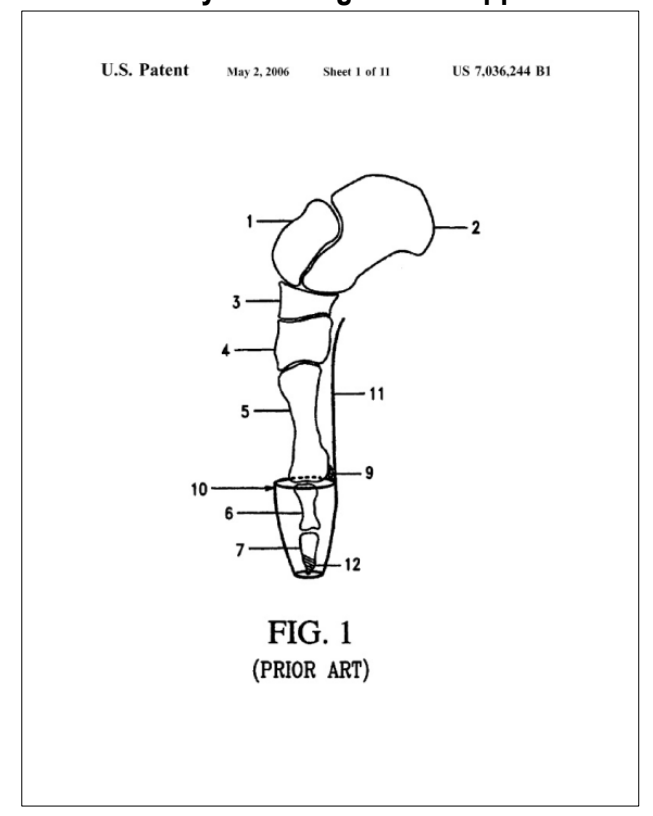

Though a toe box supports the foot, it also tightly encapsulates the foot, potentially reducing proprioceptive sense. The afferent nerves may then have reduced sensitivity, and if the box is excessively tight, it may also impede motor unit response. Dynamic balance, sensory control, and motor control are essential in equilibrium training, and a ballet dancer is trained to correct proprioceptive balance at the most inferior point possible. ${ }^{5}$ This allows for the remainder of the body to maintain control and limit postural sway, thus maintaining the illusion of effortlessness and minimizing movement in the trunk and arms. 
Several studies have been done using forceplates to assess proprioceptive balance in the body, but none have been done with pointe shoes.-12 Though the shoe itself will never likely be removed from use, there are certain innovations and techniques in fitting that may improve the care for those dancers who must continue to wear them. In an attempt to improve the employ of pointe shoes, this study explored two particular toe box styles and their effect on proprioception. There are various styles of pointe shoe toe boxes, but for the purposes of this study, two general categories were used -- the tapered box and the square box. We hypothesized that the dancer who used the tapered box pointe shoes would have poorer gains in proprioception than the dancer in square box pointe shoes, and the tapered box may have also reduced the dancer's proprioceptive sense.

\section{METHODOLOGY \\ Subjects}

Thirty-six female participants completed the study (age $=13.2+2.2$ years, height $=156.4+11.5 \mathrm{~cm}$, weight $=46.6+9.4 \mathrm{~kg}$ ). All participants were recruited from the pointe students at Life House Performing Arts Academy in Payson, Utah and Hot Shots Ballet in Pleasant Grove, Utah. Inclusion criteria were a minimum of one year of ballet training, currently enrolled in one beginning pointe class, and no pre-existing condition contraindicating the use of pointe shoes (was determined by the teacher). Students of different technique levels were divided evenly within that level, so that both groups had equal time working on and off pointe in ballet each week through the course of this study, and none were currently preparing for any upcoming shows. Prior to participation, all subjects and their parents/legal guardians signed an informed consent form approved by the university's Institutional Review Board (IRB). Participants were allowed to use and keep the pointe shoes purchased for them as their only source of compensation for participation in the study.

Subjects were assigned randomly to one of two groups. One group of 18 subjects was assigned to the tapered toe box pointe shoe, and the other group of 18 subjects was assigned to the square toe box pointe shoe. Subjects in each group were given a choice of any variation, brand, or size of shoe, only being restricted on the tapered or square box style. They were instructed to train using that shoe, exclusively, over the next six weeks. Pointe work referred to the use of pointe shoes in ballet. Regular pointe work referred to the regular class time that the student would spend on pointe in any given week, according to their technique level.

\section{Procedures}

After a minimum of 15 minutes of class, to assure that all subjects were adequately warmed up, subjects performed three trials of barefoot demi-pointe standing (on the ball of the foot) in pirouette position. Pirouette position required the dancer to stand on one leg in either full or demi-pointe, with full external rotation of both hips, the support knee in full extension and support hip in zero degrees of extension. The contralateral hip was in approximately 90 degrees of flexion and 70 degrees of hip abduction. The contralateral knee was in approximately 90 degrees of flexion and the tip of the great toe was placed on or above the knee of the support leg with the ankle in full plantar flexion and eversion (see Figure. 2). The spine was in full erect position, with all the core muscles engaged to maintain alignment and the neutrality of the pelvis. Scapulae were depressed, and the shoulders were flexed to 85 degrees. Elbows were lifted so the 20 degrees of elbow flexion ran horizontally. Forearms were fully supinated, and the wrists remained neutral. The head was placed in alignment with the spine.

\section{Figure 2. Test Position on Force Plate}

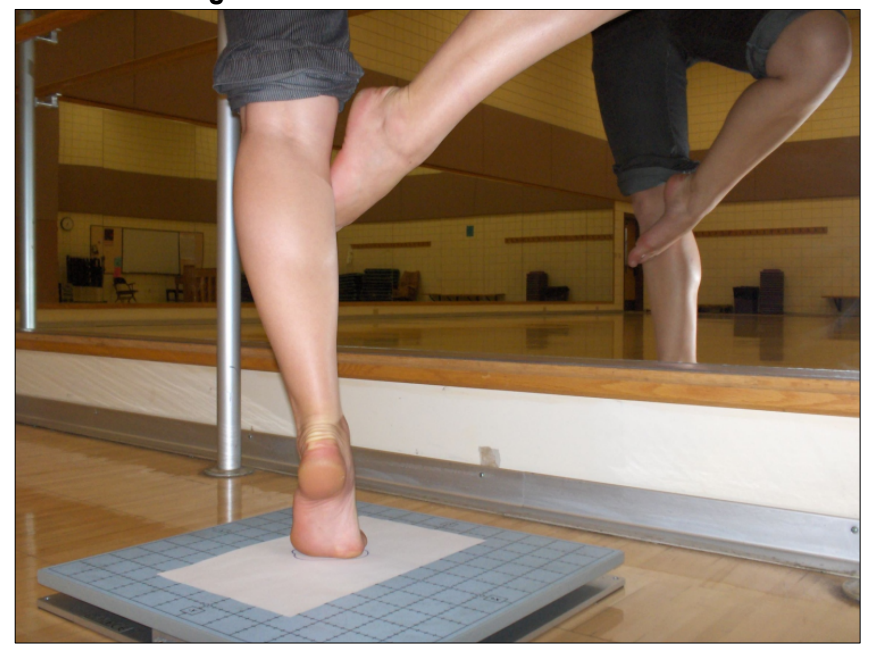


Participants were given a target to focus on, on the wall directly in front of them. They were given a moment to position themselves at the barre, and when they were ready, they lifted their arms, letting go of the barre, and the measurement was then taken for five seconds, using a forceplate (Accusway Plus, AMTI). If the subject touched down with the other foot, or took hold of the barre at any point during the recording, the trial was eliminated and they were required to repeat the trial. This trial was then repeated three times on each leg. Averages of the three trials on each leg were used for computing the statistics. The complete test was done prior to the six weeks of training and then repeated in a posttest after six weeks of training in their assigned shoe.

Proprioception measurements included the area95 referring to the area covered by the meandering COP, and Vavg referring to the velocity or average speed of the traveling center of pressure (COP) as it continues to move and change direction. These measurements were recorded by the AMTI Accusway Plus Forceplate.

\section{Instrumentation}

Proprioceptive control, under the conditions outlined above, was assessed with the Accusway Plus forceplate (AMTI, Watertown, MA). Three dimensional force and moment signals arising from the foot/forceplate interface were filtered using a fourth-order low zero lag, low-pass filter with a cutoff frequency of $5 \mathrm{~Hz}$. Center of Pressure (COP) and area 95 (area covered by the wandering COP) was calculated from the force and moment signals through Balance Clinic Software (AMTI, Watertown, MA) and sampled at a rate of $50 \mathrm{~Hz} .{ }^{11}$ These have been demonstrated to be both valid and reliable measurements in determining postural control, and thus provide an accurate estimate in determining proprioceptive deficiencies. ${ }^{8}$

Area95 represents the area of the $95^{\text {th }}$ percentile ellipse, and is calculated by the equation:

$\left.\left.\mathrm{A} \pi * \sqrt{\mathrm{F} *\left(\mathrm{x}_{\mathrm{sd}}^{2}\right.}+\mathrm{y}_{\mathrm{sd}}^{2}+\mathrm{D}\right) * \sqrt{\mathrm{F} *\left(\mathrm{x}_{\mathrm{sd}}^{2}\right.}+\mathrm{y}_{\mathrm{sd}}^{2}-\mathrm{D}\right)$. As the proprioceptors and muscles of the foot work to correct balance during stance, the COP migrates. The forceplate records the pattern of the changing COP, and the area95 equation is used to determine the variability. Thus, the smaller area generally represents greater proprioception, because the body's proprioceptive arc is working more efficiently, keeping the COP under better control. ${ }^{1}$

Vavg, which represents the average velocity of the path length per unit of time, was also calculated using the equation $\mathrm{l}_{\text {unit }}=$ $\frac{l_{\text {path }}}{\mathrm{t}} \mathrm{cm} / \mathrm{sec}$, but no statistical significances were found for this measurement. This average, if it had any significance, would represent the muscle ability to quickly correct the COP. Again, the smaller number represents greater control in proprioception. ${ }^{20}$

\section{Statistical Analysis}

The mean of the three trials on demi-pointe were determined. These mean values were then compared between the two groups, with each individual's pretest acting as their own control, and the demi-pointe measurement acting as a control between the two groups. These values were compared using a mixed design ANOVA to determine the gain or loss of proprioception in each group. The independent variable was the toe box style assigned to each group and the dependent variables were the COP and area 95 mean values for each condition.

\section{RESULTS}

There was no main effect for toe box type $\left(F_{1,32}=1.15, P=2.9\right)$. However, a Bonferroni Post-Hoc analysis showed a difference in the post-training left area95 measurement between square $\left(5.86 \pm 2.28 \mathrm{~cm}^{2}\right)$ and tapered boxes $\left(3.93 \pm 1.30 \mathrm{~cm}^{2}\right)$ $\left(F_{1,32}=3.37, P=.02\right)$. This indicated that the tapered box may have improved proprioception, because area95 represents the area covered by the center of pressure (COP), meaning the participants swayed over a smaller distance. The means and standard deviations for each condition can be found in Tables 1 and 2.

\section{Table 1. Vavg (cm/sec)}

\begin{tabular}{|l|l|l|l|l|}
\hline & \multicolumn{2}{|c|}{ Square Toe Box } & \multicolumn{2}{c|}{ Tapered Toe Box } \\
\hline & Pre & Post & Pre & Post \\
\hline Left & $5.45+1.04$ & $4.98+0.87$ & $5.50+1.43$ & $4.69+0.88$ \\
\hline Right & $5.27 \pm 1.04$ & $5.23 \pm 1.17$ & $5.44 \pm 1.43$ & $5.10 \pm 1.47$ \\
\hline
\end{tabular}

Table 2. area95 $\left(\mathrm{cm}^{2}\right)$

\begin{tabular}{|l|l|l|l|l|}
\hline & \multicolumn{2}{|c|}{ Square Toe Box } & \multicolumn{2}{c|}{ Tapered Toe Box } \\
\hline & Pre & Post & Pre & Post \\
\hline Left & $6.09+3.00$ & $5.86+2.28$ & $5.90+2.54$ & $3.93 \pm 1.30$ \\
\hline Right & $6.60 \pm 2.36$ & $5.31+1.98$ & $6.06+2.70$ & $5.01+2.60$ \\
\hline
\end{tabular}




\section{DISCUSSION}

No significant differences were found between the groups on any of the measurements, except the area95 measurement for the left foot. The findings suggest that proprioception was somewhat improved by wearing the tapered shoe, contrary to our hypothesis. However, this change did not occur in both feet and may have been caused by order of testing (left was always tested first) and/or other extraneous variables. One such variable is related to proper warm-up. Only ten to fifteen minutes of warm-up were permitted for the first group of participants, while others had sometimes considerably more. As time was limited, and it took about two and half hours to test only 20 subjects, it was impossible to give them equal warm-up, and we did not account for the order in which the participants were tested. There was a noted trend in all conditions of improved proprioception, although most were not statistically significant. This was an expected training effect of their six week training program on pointe.

After the first ten pre-tests, the protocol was changed from measuring both demi-pointe and full pointe in the respective shoes assigned to each group, to measuring only demi-pointe barefoot for everyone. This change came about due to time constraints and the fact that some of the first girls were having a difficult time on full pointe, even for five seconds. It was determined that because the study was seeking a long-term effect from specific toe boxes on the dancer's proprioception, the same results could be measured with everyone on demi-pointe as proprioceptive muscular deficiencies could still be detected. Whether the results would have been different, had we measured the girls on full pointe as well, is therefore unanswered. Furthermore, most of the subjects dance jazz and/or other styles throughout the week, allowing them time in flat shoes and barefoot dancing. With only one to five hours of pointe work each week, these other dance styles could easily have counteracted the effect that the pointe shoes may have had on the girls' feet. Other limitations may include the many other variations in shoe style according to preference in style, brand, vamp, width, side wings, shank, depth and more. Genetic variations, motivation of the dancers, current health, and/or pre-existing conditions not identified at the time of measurement may have also played a role in unanticipated results.

Future research should include measuring the pre- and post-tests in the assigned pointe shoes. It may also be beneficial to focus only on girls who have at least one or two years of training on pointe. This would make the testing much quicker and easier. Girls with less training tend to have a hard time balancing for even five seconds. For the pre-test, a few girls failed to complete all of the trials and their data could not be used in this study. However, for the post-test only six weeks later, all the subjects were able to complete every trial. Future studies should also be conducted for a longer period of time, as detrimental effects of pointe shoes may take longer to develop.

Future research should also evaluate specific injuries associated with particular shoes. A retrospective study could be used to discover what types of injuries and/or imbalances may have occurred as a result of either toe box. Narrowing the range of shoe styles may also be an option. This study placed all shoes within the two categories of tapered and square, but there are many variations within these styles. By allowing the girls to choose any shoe within their assigned category, it may be that the options were too broad.

\section{ACKNOWLEDGMENTS}

The authors would like to thank Dr. Timothy Uhl, at the University of Kentucky, for his assistance with the data analysis.

\section{REFERENCES}

1. Paparizos AL, Tripp DA, Sullivan MJL, Rubenstein ML. Catastophizing and Pain Perception in Recreational Ballet Dancers. Journal of Sport Behavior. 2005;28.

2. Pickard A. Schooling the dancer: the evolution of an identity as a ballet dancer. Research in Dance Education. 2012;13(1):25-46.

3. Houglum PA. Therapeutic Exercise For Musculoskeletal Injuries. Second ed. Champaign: Human Kinetics; 2005.

4. Guillou E, Dupui $P$, Golomer E. Dynamic balance sensory motor control and symmetrical or asymmetrical equilibrium training. Clinical Neurophysiology. 2009;118(2):317-24. [PMID 17140847]

5. Lee AJ, Lin WH. Twelve-week biomechanical ankle platform system training on postural stability and ankle proprioception in subjects with unilateral functional ankle instability. Clinical Biomechanics [serial online]. October 2008;23(8):1065-72. [PMID 18621453]

6. Windhorst U. Muscle proprioceptive feedback and spinal networks. Brain Res Bull. 2007 Jul 12;73(4-6):155-202. [PMID 17562384]

7. Ergen E, Ulkar B. Proprioception and Coordination. In: Frontera WR, Micheli LJ, Herring SA, Silver JK, eds. Clinical sports medicine: medical management and rehabilitation. Philadelphia, PA: Saunders; 2007:237-8.

8. Hertel J, Olmsted-Kramer LC. Deficits in time-to-boundary measures of postural control with chronic ankle instability. Gait Posture. 2007;25(1):33-9. [PMID 16446093] 
9. Hertel J, Olmsted-Kramer LC, Challis JH. Time-to-boundary measures of postural control during single leg quiet standing. Journal of Applied Biomechanics. 2006;22(1):67-73. [PMID 16760569]

10. McKeon PO, Hertel J. Plantar hypoesthesia alters time-to-boundary measures of postural control. Somatosensory \& Motor Research. 2007;24(4):171-7. [PMID 18097990]

11. McKeon PO, Hertel J. Spatiotemporal postural control deficits are present in those with chronic ankle instability. BMC Musculoskeletal Disorders. 2008;9:76. [PMID 18518994]

12. McKeon PO, Ingersoll CD, Kerrigan DC, Saliba E, Bennett BC, Hertel J. Balance training improves function and postural control in those with chronic ankle instability. Medicine And Science In Sports And Exercise. 2008;40(10):1810-9. [PMID 18799992]

13. Gaines $\mathrm{D}$. The relative effectiveness and correct sequencing of proprioceptive neuromuscular facilitation techniques (PNFT) and active rockerboard exercises in the rehabilitation of chronic ankle sprains. [M. Tech Chiropractic]: Department of Chiropractic, Durban Institute of Technology; 2005.

14. Butler AA, Lord SR, Rogers MW, Fitzpatrick RC. Muscle weakness impairs the proprioceptive control of human standing. Sydney and Chicago: Prince of Wales Medical Research Institute and University of New South Wales Department of Physical Therapy and Human Movement Science. Northwestern University, Feinberg School of Medicine; 2008.

15. Franklin E. Dynamic Alignment Through Imagery. Champaign: Human Kinetics; 1996.

16. Fitt SS. Dance Kinesiology, 2nd edition. Salt Lake City: Schirmer Books; 1996.

17. Sherwood L. Human Physiology From Cells to Systems, 6th ed. Belmont: Thompson Books/Cole; 2007.

18. Baechle TR, Earle RW. Essentials of Strength Training and Conditioning, 3rd ed. Champaign: Human Kinetics; 2008.

19. Finch D. Inventor: Rigid Articulated Pointe Shoe. US patent 7036244. May 2, 2006.

20. Balance Clinic. Balance Software for AMTI's Accusway Plus Balance Platform. Vol Watertown: AMTI; 2006. 